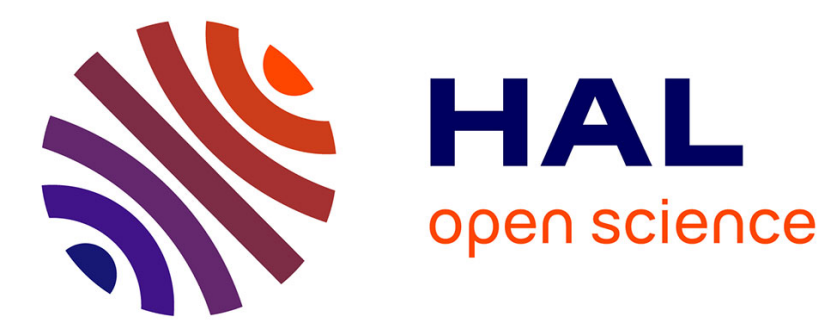

\title{
Calibration based on part set-up measurement for on-machine inspection using vision
}

Lorène Dubreuil, Yann Quinsat, Claire Lartigue

\section{To cite this version:}

Lorène Dubreuil, Yann Quinsat, Claire Lartigue. Calibration based on part set-up measurement for on-machine inspection using vision. International Journal on Interactive Design and Manufacturing, 2015, pp.1-7. 10.1007/s12008-015-0290-3 . hal-01194565

\section{HAL Id: hal-01194565 \\ https://hal.science/hal-01194565}

Submitted on 7 Sep 2015

HAL is a multi-disciplinary open access archive for the deposit and dissemination of scientific research documents, whether they are published or not. The documents may come from teaching and research institutions in France or abroad, or from public or private research centers.
L'archive ouverte pluridisciplinaire HAL, est destinée au dépôt et à la diffusion de documents scientifiques de niveau recherche, publiés ou non, émanant des établissements d'enseignement et de recherche français ou étrangers, des laboratoires publics ou privés. 


\title{
Calibration based on part set-up measurement for on-machine inspection using vision
}

\author{
Lorène Dubreuil · Yann Quinsat · Claire Lartigue
}

Received: date / Accepted: date

\begin{abstract}
Integrating inspection procedures in the machining process contributes to process optimization. To be highly efficient, inspection operations must be performed in the same frame as machining operations. Within the context of on-machine inspection using vision, a method of calibration based on part set-up measurement is proposed in this paper. The measurement frame thus coincides with the CAD/CAM frame in which the CAD model is built. The CAD-based calibration is performed by using $3 \mathrm{D}$ features of the part set-up, which are easily identifiable in the 2D picture of the scene. The robustness of the method with regard to $2 \mathrm{D}$ feature identification in real environment is assessed thanks to Monte-Carlo simulations.
\end{abstract}

Keywords CAD-based Calibration - On-machine inspection · Vision

\section{Introduction}

The integration of inspection procedures in the production process contributes to the improvement of the manufacturing process. Associated benefits include high speed of inspection, measurement flexibility, on-machine inspection, and the possibility of $100 \%$ inspection [1]. On-machine inspection (or in-situ inspection) is performed while the part is still located on the machine tool and while the machining process is stopped [2]. On-machine inspection allows to achieve high productivity, to reduce lead times, and to make a rapid decision regarding the conformity of the produced part [3]. Furthermore, due to the great inter-operability between machining and inspection, in-situ inspection, either for dimen-

C. Dubreuil · Y. Quinsat · C. Lartigue

LURPA, ENS Cachan, Univ Paris-Sud, F-94235 Cachan, France

Tel.: +33-1-47402986

Fax: +33-1-47402200

E-mail: quinsat@lurpa.ens-cachan.fr

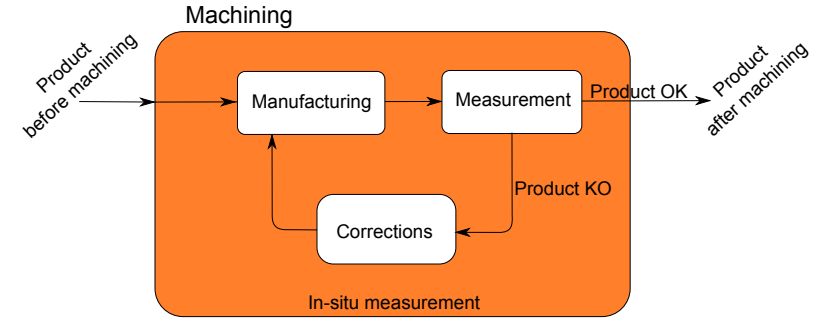

Fig. 1 On-machine inspection using vision

sional metrology or for surface roughness, contributes to process optimization [4], [5], [6].

On-machine inspection is often performed using vision. Vision-based systems are used for the verification of machining set-ups [7], [8], the survey of tool trajectories [9], part inspection of socket production, and so on [1]. These systems present various advantages: low cost, rapidity, flexibility and simplicity of implementation in the context of production. A minor drawback is the system calibration. Calibration is necessary to transform a $2 \mathrm{D}$ point of the picture into a 3D point expressed in the reference frame. Within the context of on-machine inspection, the positioning of the picture frame relatively to the reference frame must be performed for each measurement. On the other hand, measurement and machining operations must be performed in the same frame to facilitate the comparison of the machined part to its CAD model. Therefore, to enhance the interoperability between machining and inspection, it could be interesting that the reference frame used for calibration coincides with the CAM frame associated to machining operations. This removes an additional step of frame registration which could be penalizing in terms of computational time and quality. The idea is thus to build the reference frame from features belonging to the machine tool scene. In this direction the use of features belonging to the part set-up seems relevant. This paper deals with a calibration method based on part set- 
up measurement for on-machine inspection. In the proposed approach, features belonging to the part set-up are used to map the vision calibration frame to the machining frame in which both measurements and machining operations will be performed. The final aim of our study is to develop a tool for manufacturing monitoring with the aim of improving the interoperability between CAM, machining and inspection during the production start of a new part. This tool based on vision (more particularly image correlation) performs onmachine part inspection at the beginning of the machining in order to locate potential defects (Fig. 1). It thus gives the user a rapid and interactive decision tool to act at the earliest to make corrections. The paper is organized as follows. Section 2 is dedicated to the basics of calibration from setup measurements. Applications of our approach are detailed in section 3. In section 4, a sensitivity study is proposed to assess the robustness of the method with regard to environment parameters.

\section{Calibration from set-up measurements}

Before detailing our approach, let us review the basic principle of vision-system calibration along with classical methods proposed in the literature.

\subsection{Calibration principle}

The calibration aims at identifying the model parameters that define the relationship between the coordinates of a $2 \mathrm{D}$ point, expressed in the picture $R_{p}$, and the coordinates of a 3D point expressed in the reference frame $R_{w}$.
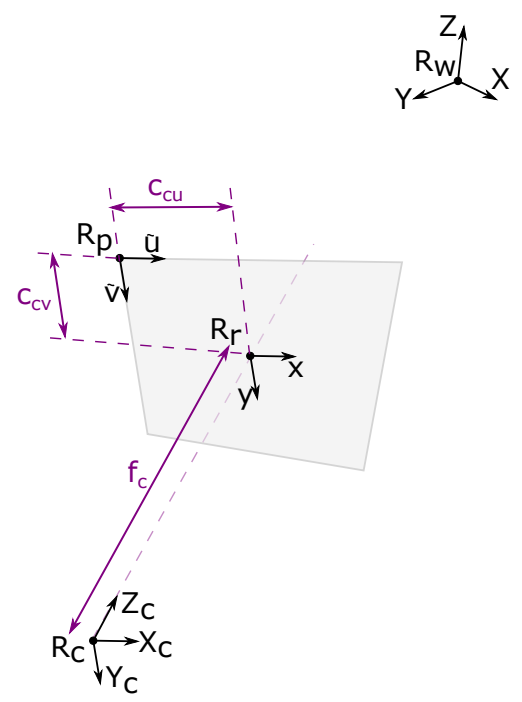

Fig. 2 Pinhole model

$$
\left(\begin{array}{c}
u \\
v \\
1
\end{array}\right)_{R_{p}}=A \cdot D \cdot P \cdot T \cdot\left(\begin{array}{c}
X \\
Y \\
Z \\
1
\end{array}\right)_{R_{w}}=M \cdot T \cdot\left(\begin{array}{c}
X \\
Y \\
Z \\
1
\end{array}\right)_{R_{w}}
$$

Based on the pinhole model (Fig. 2), the relationship between $R_{p}$ and $R_{w}$ results from the combination of the geometric transformations $A, D, P$, and $T$ as defined in (Eq. 1). The matrix $A$ corresponds to the affine transformation from the "center" picture frame $R_{r}$ to the picture frame $R_{p}$. The transformation $P$ is a perspective projection of $R_{c}$, the frame attached to the camera, into the retinal plane $R_{r}$. The transformation $D$ corresponds to camera distortions. The parameters associated with the matrices $A, D$, and $P$ correspond to the internal calibration parameters also called the intrinsic parameters: the focal $f_{c}$, the picture center position $C_{c u}$ and $C_{c v}$, the pixel size $s_{p}$, the pixel number $n_{p}$, and the distortion parameters $k_{i}$ (for $\mathrm{i}=1$ to 5 ).

The transformation $T$ between the reference frame $R_{w}$ and the camera frame $R_{c}$, corresponds to the combination of a rotation $R(3 * 3)$ and of a translation $t$. $T$ can be described thanks to a homogeneous matrix, and its parameters are called extrinsic parameters (Eq. 2).

$\left(\begin{array}{c}X_{c} \\ Y_{c} \\ Z_{c} \\ 1\end{array}\right)_{R_{c}}=T \cdot\left(\begin{array}{c}X \\ Y \\ Z \\ 1\end{array}\right)_{R_{w}}=\left(\begin{array}{cc}R_{33} & t \\ 0 & 1\end{array}\right) \cdot\left(\begin{array}{c}X \\ Y \\ Z \\ 1\end{array}\right)_{R_{w}}$

Several techniques of calibration exist. Most of them allow the determination of extrinsic and intrinsic parameters simultaneously. Starting from a correspondence between 2D to $3 \mathrm{D}$ features, classical techniques rely on the minimization of a projection error function [10][11][12]. Several methods are based on point correspondence. Faugeras and Toscani [11] propose a method leading to the identification of the pinhole model parameters without distortions. As they only consider the just necessary number of points, the problem is linear and easy to solve. Lavest et al. [12] extend the approach by considering a non-limited number of points. The correspondence problem is thus non-linear, and is solved by minimizing projection errors. This method has been implemented as a Matlab R2014 (c) toolbox by Bouguet [13]. Douilly [14] propose a calibration method for a multi-camera system based on a ball-bar artifact. Inspired from the method developed by Tsai [15], this approach requires a high number of pictures to be efficient. A second family of methods use line correspondences. The difference lies in the model used to described the lines: parametric description in [10], or plane intersection in [16]. A few studies rely on the CAD definition of the studied scene to perform the calibration. Beaubier et al. [17] proposed a CAD-based calibration using stereo-correlation. Unfortunately, this approach, which 


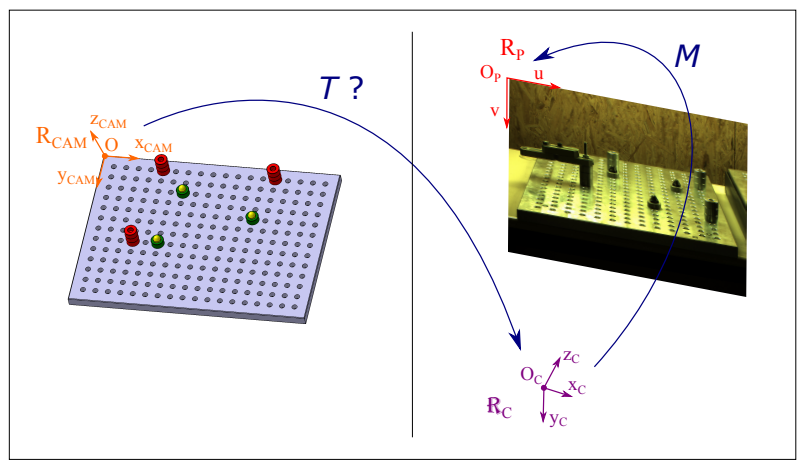

Fig. 3 Identification of the transformation $T$

takes place during machining, relies on the part which has geometrical defects. Furthermore, the stereo-correlation requires a pattern projection which is not appropriate for onmachine inspection due to low contrast, the difficulty to extract more than a few features, and it may be time consuming.

\subsection{Proposed approach}

As the vision system is dedicated to on-machine inspection, the calibration method proposed in this paper relies on identifiable geometric features of the machine tool scene. As aforementioned, the idea is to define a unique frame for calibration, vision-based measurements, and machining operations. In this direction, we propose that the reference frame $R_{w}$ coincides with the frame in which machining operations are defined. Machining operations are generally defined in a frame associated to the CAD model. It thus seems relevant to calibrate the system thanks to features that belong to the machining set-up as they belong to both the CAD model and the machining environment, and as they are also not modified during machining. This will make the integration of the measurement in the machining process easier.

During on-machine vision, intrinsic parameters are fixed and do not vary. Therefore, the transformation $M=A . D . P$ between $R_{p}$ and $R_{c}$ is completely defined, and the study focuses on the identification of the extrinsic parameters i.e. on the identification of the elements of the transformation matrix $T$ (Fig. 3). The method we propose aims at establishing the correspondence between a set of 3D features of set-up CAD model and the corresponding 2D features in the picture of the actual machining set-up. Once the correspondence is established, the transformation $G$ between $R_{p}$ and $R_{w}=R_{C A M}$ is known, and $T$ is simply given by:

$T=M^{-1} \cdot G$

It is important to underline that the set-up defects are neglected: the actual machining set-up is assimilated to its
Table 1 Features identifiable on pictures

\begin{tabular}{lcc}
\hline 3D feature & 2D feature & Extracted element \\
\hline \hline Cylinder & 2 generatrices & Line \\
Sphere & Circle & Point \\
Intersection of 2 planes & Line & Line \\
Circle & Ellipse & Point \\
\hline
\end{tabular}

CAD model. The idea is thus to use features that can be easily identifiable as displayed in Tab. 1.

Table 1 describes the correspondence between a 3D geometrical feature, the associated $2 \mathrm{D}$ element belonging to the picture, and the extracted 2D element. The transformation $T$ is characterized by 6 extrinsic parameters, 3 Euler angles $(\phi, \theta, \psi$ for the rotation $R(3 * 3))$ and 3 distances for the translation $t$. Let us denote $\Phi=\left(\phi, \theta, \psi, t_{x}, t_{y}, t_{z}\right)$ the vector gathering the extrinsic parameters we want to identify. The set of features must lead to a minimum of 6 independent equations to completely define the transformation.

The method consists in 3 main steps. An initial vector $\Phi_{0}$ is chosen defining an initial transformation $T_{\text {init }}$. The 3D features of the CAD model are projected onto the picture frame thanks to equation 1 (Fig. 4(a)). In parallel, equivalent 2D features are extracted from the picture (Fig. 4(b)). To illustrate our purpose, let us consider the case of the point $\mathrm{C}$ belonging to the CAD model. $\mathrm{C}$ is projected onto the picture frame $R_{p}$ in $C^{\prime}$ by the combination of the transformation $T_{\text {init }}$ and the internal transformation $M$. The same goes for the line $d_{C A D}$ belonging to the CAD model which is projected in $d_{\text {pict }}$ into the picture frame. In parallel, corresponding elements are extracted from the picture: the point $\mathrm{D}$ and the line $d_{\text {detect }}$ (Fig. 4(b)). It is thus possible to calculate the projection error for each type of feature. For points, the error is defined as the distance in pixels between the extracted point and the projected point as represented in figure 5.

For lines, two errors are calculated corresponding to the distances of the most extreme points of the projected line $d_{\text {pict }}$ to the extracted line $d_{\text {detect }}$ (Eq. 4).

$\left\{\begin{array}{l}e_{\text {point }}=d\left(C^{\prime}, D\right) \\ e_{\text {axis }}{ }^{1}=d\left(A^{\prime}, d_{\text {detect }}\right) \\ e_{\text {axis }}{ }^{2}=d\left(B^{\prime}, d_{\text {detect }}\right)\end{array}\right.$

For a set of $\mathrm{m}$ lines and $\mathrm{n}$ points, this yields to an error vector $E(\Phi)$ of dimension $2 \mathrm{~m}+\mathrm{n}$. The matching problem between $3 \mathrm{D}$ features and $2 \mathrm{D}$ features, i.e. the calibration, leads to the minimization of the function $F=E^{T} \cdot E$. A first-order expansion of the error near the initial point $\Phi_{0}$ is performed:

$E(\Phi)=E\left(\Phi_{0}\right)+\left[\frac{\partial E}{\partial \Phi}\right]_{\Phi_{0}}$ 


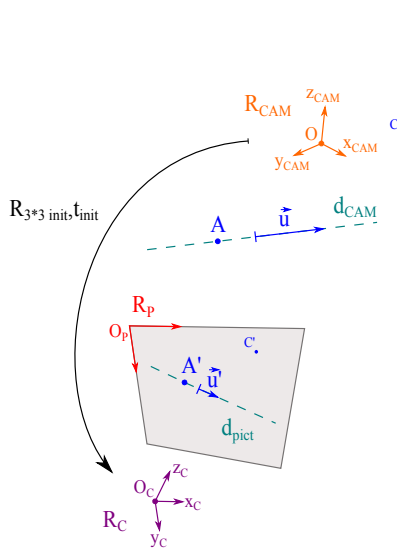

(a) 3D feature projection

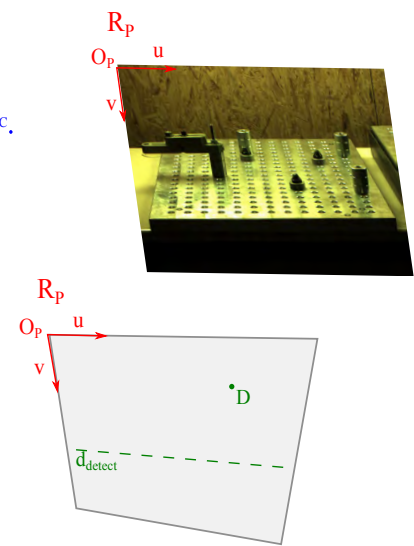

(b) 2D feature extraction

Fig. 4 Main steps of the approach

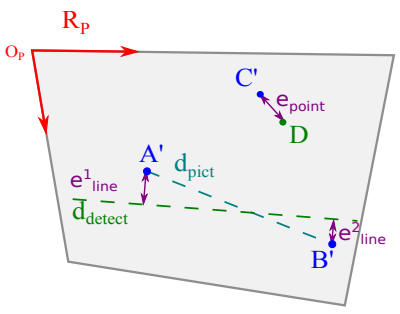

Fig. 5 Calculation of the projection error

Let us denote $J=\left[\frac{\partial E}{\partial \Phi}\right]_{\Phi_{0}}$ the jacobi of $E$. The problem is thus to find $\Phi$ so that $F$ is minimized. This gives:

$\left[\frac{\partial F}{\partial \Phi}\right]=2 \cdot E^{T} \cdot\left[\frac{\partial E}{\partial \Phi}\right]=0$

Considering the Jacobi of $\mathrm{E}$, this yields to:

$J^{T} \cdot E=0$

Then:

$J^{T} \cdot E=J^{T} \cdot\left(E\left(\Phi_{0}\right)+J \cdot d \Phi\right)=J^{T} \cdot E\left(\Phi_{0}\right)+J^{T} \cdot J \cdot d \Phi=0$

And finally:

$d \Phi=-\left(J^{T} \cdot J\right)^{-1} \cdot\left(J^{T} \cdot E\left(\Phi_{0}\right)\right)$

The problem is solved using Matlab (c) pseudoinverse. If the transformation $T_{\text {init }}$ is close enough to the optimized solution, the problem can be solved with only one iteration. If not, the optimization is reiterated considering that the initial transformation is given by $\Phi_{1}=\Phi_{0}+d \Phi$.

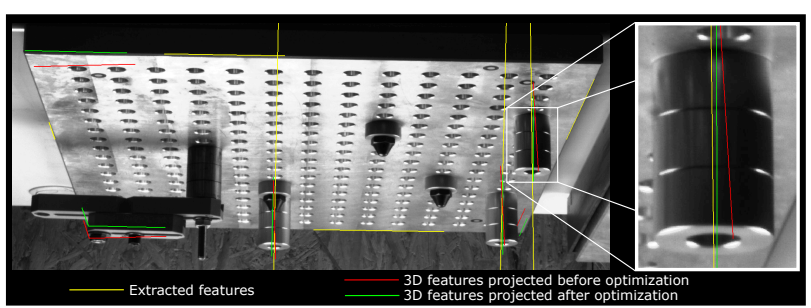

Fig. 6 CAD-based calibration

\subsection{Validation of CAD-based calibration}

The approach is applied to the set-up presented in figure 3. First, the intrinsic calibration is performed using the Camera Calibration toolbox of Matlab (c) [13]. Intrinsic parameters are identified and the matrix $M$ is known. The initial transformation $T_{\text {init }}$ is defined considering the manual matching of 6 points defined in the CAD model to the corresponding points on the picture. 3D features are projected onto the picture according to equation 1 , giving elements in red in figure 6.

In parallel, feature extraction from the picture is performed using the toolbox Image Processing of Matlab (c). After filtering the picture using the Canny filter, features are extracted from the picture thanks to the Hough transform. When necessary, the extracted element is constructed as it is the case for axis cylinders (see figure 7). Extracted features are reported in yellow in figure 6 . The error function is thus calculated and the optimization problem is solved thanks to the least-square method. Results are displayed in figure reffig:validation, in which the green elements are the optimized calculated features. As it can be observed, positions of the optimized features are close to the nominal ones, assessing the relevance of CAD-based calibration.

Nevertheless, there are still deviations between the projected and the extracted features due to 2 main causes. The first one is that the set-up, which is assumed to be perfect, presents some defects that should be taken into account. Dimensions and positions of the components belonging to the set-up do not perfectly match their configuration of the CAD model. On the other hand, the method used to extract $2 \mathrm{D}$ features from the picture involves some errors. The extraction is dependent on the quality of the picture along with the parameters associated to the extraction method. Both sources of er-
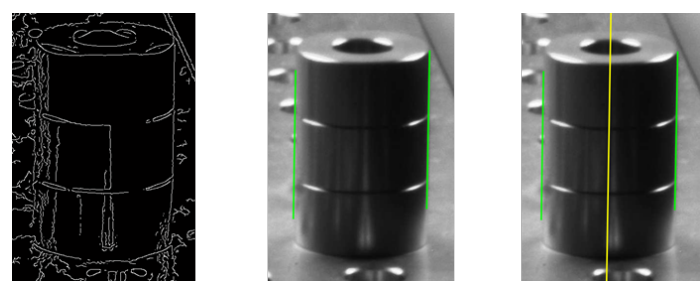

Fig. 7 2D feature extraction 


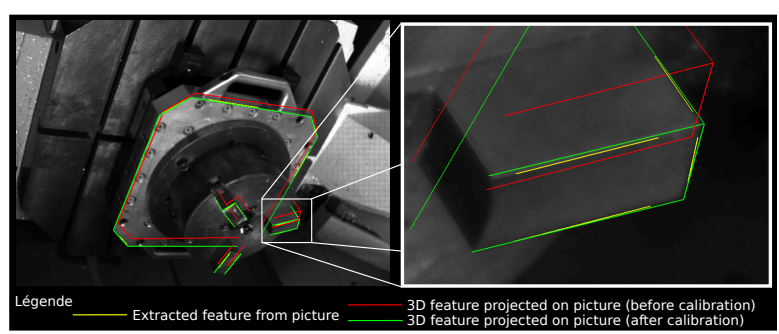

Fig. 8 Calibration from set-up measurement in real environment

rors have the same importance on the deviations. In order to quantify the uncertainty associated to the proposed method, the influence of 2D feature extraction is investigated in the next section.

\section{Application and sensitivity study}

\subsection{Description of the case study}

The influence of 2D feature extraction is investigated considering a real case for which the set-up is in situation in the machine tool environment. The calibration is performed at the beginning of the production, problems of coolants or removed chips/strings obscuring vision views are avoided. The approach is applied by considering a set of 17 lines to perform the calibration (Fig. 8). To investigate errors associated to the calibration, the projection error is calculated for a set of points belonging to the set-up. These points, which correspond to circle centers, were not used for calibration (Fig. 9).

Table 2 Reprojection errors

\begin{tabular}{ccc}
\hline & Mean (pixel) & Standard deviation (pixel) \\
\hline$e_{u}$ & -0.282 & 3.688 \\
$e_{v}$ & 0.234 & 3.058 \\
\hline
\end{tabular}

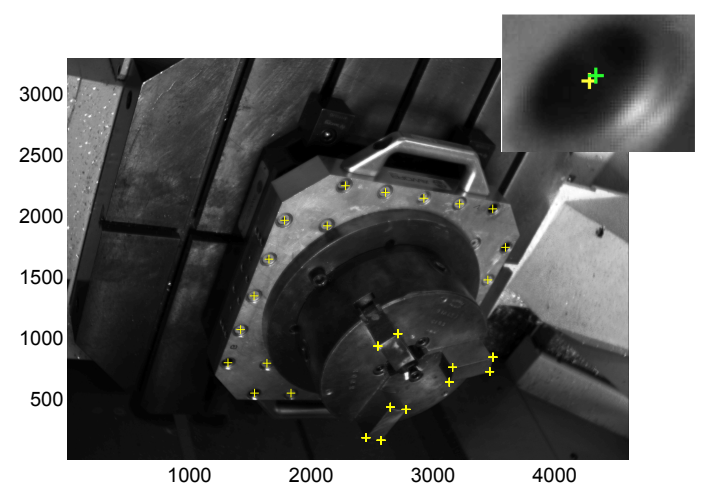

Fig. 9 Projection errors for a set of points
Results reported in table 2 enhance the quality of the calibration. Actually, the absolute mean value of the reprojection error does not exceed 0.24 pixel for both direction $u$, and $v$. Taking into account the dimensions of the measured space $(710 \mathrm{~mm} \times 710 \mathrm{~mm})$, related to the image size (3200 $\times 4500$ pixels), the error is consistent with the order of magnitude of the machining defects we want to evaluate. Nevertheless, one of the key points of using this method for on-machine inspection deals with its sensitivity to feature extraction and changes in calibration conditions. A sensitivity study is investigated in the next section.

\subsection{Sensitivity of the proposed method}

The sensitivity study is conducted by considering variability in position and orientation of the extracted 2D features. A 2D line is defined in the picture by its explicit model, $y=a \cdot x+b$, where $a$ represents the slope and $b$ the intercept. An uncertainty is thus associated to each parameter $a$ and $b$, and is modeled by a Gaussian distribution characterized by a standard deviation $\delta a$, and $\delta b$ respectively. $\delta a$, and $\delta b$ are obtained by considering the variations in position and orientation of a set of extracted lines for different calibrations for which lighting conditions are modified along with the size of the area around the feature to be extracted. Indeed, lighting conditions can vary widely in the machine tool environment, which can affect the quality of the $2 \mathrm{D}$ picture, and as a result, can influence 2D feature extraction. The size of the picture area from which the 2D feature is extracted also plays a role in the quality of feature's extraction. On the other hand, it is necessary to account for the variability of the intrinsic parameters, which also could affect the global calibration. These intrinsic parameters obtained using the the Camera Calibration toolbox of Matlab (c) [13] and their standard deviations are displayed in table 3. An uncertainty is then affected to each intrinsic parameter, which is modeled by a Gaussian distribution characterized by its standard deviation. Once uncertainties are affected to each parameter to account for its variability, the uncertainty associated with CAD-based calibration is obtained thanks to 1000 Monte-Carlo simulations (Fig. 10).

The sensitivity study is conducted by considering the influence of the variability of all the parameters on the one hand, and on the other hand, by only taking into account the influence of the 2D feature extraction. Results, reported in figures 11 and 12, bring out that the standard deviation of the reprojection errors do not exceed 4.317 pixels for $\sigma_{e_{u}}$ and 4.211 pixels for $\sigma_{e_{v}}$ in the second case. These values are twice lower than those obtained when all sources of uncertainty are taken into account. This observation also applies to mean error values. These results enhance the good stability of the CAD-based calibration method with regard to $2 \mathrm{D}$ feature extraction. Note that, a part of the error is likely due 


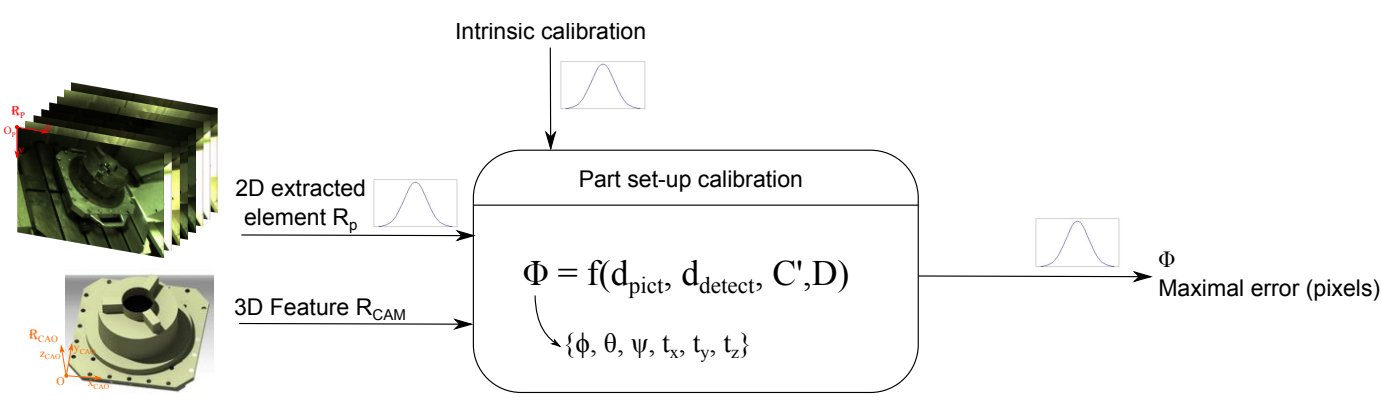

Fig. 10 Monte carlo description

Table 3 Calibration parameters

\begin{tabular}{lcc}
\hline & Mean & Standard deviation \\
\hline$f_{c} \cdot \delta_{u}$ & 6596.9 & 11.9 \\
$f_{c} \cdot \delta_{v}$ & 6583.4 & 12.0 \\
$C_{c u}$ & 2332.1 & 16.3 \\
$C_{c v}$ & 1744.13 & 16.94 \\
$k_{1}$ & -0.245 & 0.003 \\
$k_{2}$ & 0 & 0 \\
$k_{3}$ & $5.8 e^{-4}$ & $4.2 e^{-4}$ \\
$k_{4}$ & $18 e^{-4}$ & $3.9 e^{-4}$ \\
$k_{5}$ & 0 & 0 \\
\hline
\end{tabular}

to the set-up defects: dimensions and positions of the features belonging to the actual set-up do not perfectly match their configuration in the CAD model. As a conclusion, the variation in lighting conditions does not have more influence on the reprojection error than the variability of the intrinsic parameters, which appears to be significant in the uncertainty budget. This confirms that calibration based on measurements of features belonging to the part set-up is relevant for on-machine inspection using vision. Nevertheless, some key issues still need to be addressed such as improvement of intrinsic calibration, and consideration of the actual set-up.

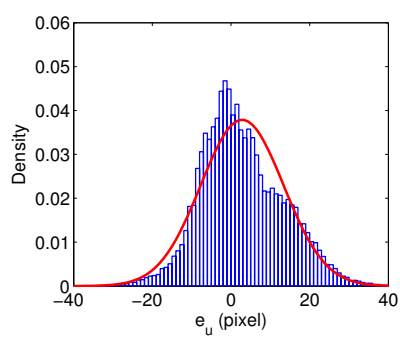

(a) $\overline{e_{u}}=2.828 \sigma_{e_{u}}=10.529$

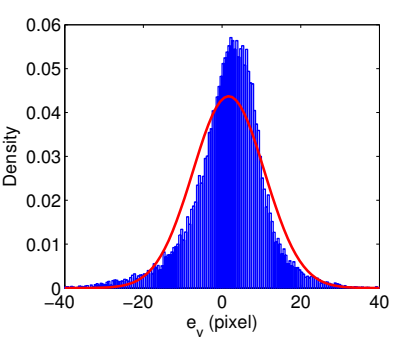

(b) $\overline{e_{v}}=1.682 \sigma_{e_{v}}=9.127$
Fig. 11 Distribution of reprojection errors considering all sources of uncertainty

\section{Conclusion}

In this study, we presented a CAD-based calibration method Based on the CAD model of a machining set-up, this new technique is particularly well-adapted for on-machine part inspection, as it allows measurements in the manufacturing frame. It relies on the matching of 3D CAD-features of the machining set-up with corresponding features extracted from the 2D image. The first results show the relevance of the proposed approach, attested by the good stability of the method with regard to image analysis. Future works will focus on the following stage of on-machine measurement for part inspection using vision. This stage deals with the implementation of a correlation-based method to localize geometrical defects by vision.

\section{References}

1. Chen, F. and Su, C., Vision-based automated inspection system in computer integrated manufacturing, The International Journal of Advanced Manufacturing Technology, 11, pp 206-213, (1996)

2. Vacharanukul, F., Mekid, S., In-process dimensional inspection sensors, Measurement, 38, pp 204-218, (2005)

3. Yandayan, T., Burdekin, M., In-process dimensional measurement and control of workpiece accuracy, Internationa Journal of Machine Tool and Manufacturing, 37(10) pp 1423-1439, (1997)

4. Quinsat, Y., Tournier, C., In situ non-contact measurements of surface roughness, Precision Engineering, 36, pp 97-103, (2012)

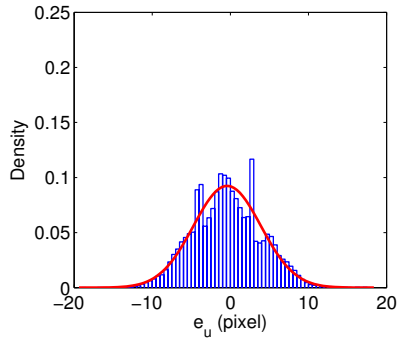

(a) $\overline{e_{u}}=-0.407 \sigma_{e_{u}}=4.317$

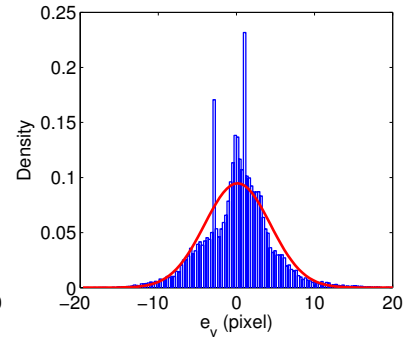

(b) $\overline{e_{v}}=0.139 \sigma_{e_{v}}=4.211$
Fig. 12 Distribution of reprojection errors considering 2D feature extraction variability only 
5. Poulahon, F.,Génération en ligne de trajectoires d'usinage par une approche de réduction de modéle, $\mathrm{PhD}$ thesis, Ecole Centrale de Nantes, (2015)

6. Poulhaon, F., Rauch, M., Leygue, A., Hascoet, J.Y., Chinesta, F.,Online Prediction of Machining Distortion of Aeronautical Parts Caused by Re-Equilibration of Residual Stresses, Key Engineering Materials vol. 612, pp1327-1335 (2014)

7. Karabagli, B, Vérification automatique des montages d'usinage par vision : application la sécurisation de l'usinage, $\mathrm{PhD}$ thesis, Université de Toulouse Le Mirail, 6 novembre 2013

8. Karabagli, B., Simon T., Orteu J.,J. Computer-vision-based automatic verification of machining setups: method for automatic pose estimation Joint International Conference of ISEM-ACEM-SEM-7th ISEM'12, Taipei (Taiwan) 11 november 2012

9. Ahmad, R., Tichadou, S., Hascoet, J.-Y., Integration of vision based image processing for multi-axis $\mathrm{CNC}$ machine tool safe and efficient trajectory generation and collision avoidance, Journal of Machine engineering, vol. 10, pp53-65, (2010)

10. Dornaika, F., Garcia, C., Robust camera calibration using 2D to 3D feature correspondences, Proceedings of the International Symposium SPIE Optical Science Engineering and Instrumentation, Videometrics V, vol. 3174, pp 123-133, (1997)

11. Faugeras, O., Toscani, G., Camera calibration for 3D computer vision, International Workshop on Machine Vision and Machine Intelligence, pp 240-247, (1987)

12. Lavest, J.-M., Viala, M., Dhome, M., Do we really need an accurate calibration pattern to achieve a reliable camera calibration?, Computer Vision ECCV98, vol. 1406, pp 158-174, (1998)

13. Bouguet, J.Y. "Camera Calibration Toolbox for Matlab", Computational Vision at the California Institute of Technology. (2010)

14. Douilly, M., Contribution lamélioration de la qualit gomtrique de positionnement dun robot anthropomorphe dans le cas dassemblage de structures aronautiques, $\mathrm{PhD}$ thesis, Ecole Normale Supérieur de Cachan, 24 mars 2009

15. Tsai.R. Y., An Efficient and Accurate Camera Calibration Technique for 3D Machine Vision, Proceedings of IEEE Conference on Computer Vision and Pattern Recognition, Miami Beach, FL, pp 364374, (1986)

16. Ma, S., Wei,G.,Huang J., Segment based camera calibration. Journal of Computer Science and Technology, vol. 8(1), pp 11-16, (1993)

17. Beaubier, B., Dufour, J.-E., Hild, F., Roux, S., Lavernhe, S., Lavernhe-Taillard, K., CAD-based calibration and shape measurement with stereodic, Experimental Mechanics, vol. 54, pp 329-341, (2014) 